\title{
Human care system for heart-rate and human-movement trajectory in home and its application to detect mental disease
}

Yutaka Hata, Seigo Kanazawa, Maki Endo, Naoki Tsuchiya, Hiroshi Nakajima

Yutaka Hata, Seigo Kanazawa, Maki Endo, Naoki Tsuchiya, Hiroshi Nakajima, "Human care system for heart-rate and human-movement trajectory in home and its application to detect mental disease," Proc. SPIE 8401, Independent Component Analyses, Compressive Sampling, Wavelets, Neural Net, Biosystems, and Nanoengineering X, 840119 (10 May 2012); doi: 10.1117/12.918552

Event: SPIE Defense, Security, and Sensing, 2012, Baltimore, Maryland, United States 


\title{
Health Care System for Heart Rate and Human Movement Trajectory in House and Its Application to Detect Mental Diseases
}

\author{
Yutaka Hata $^{\mathrm{a}, \mathrm{b}}$, Seigo Kanazawa ${ }^{\mathrm{a}}$, Maki Endo $^{\mathrm{c}}$ and Naoki Tsuchiya ${ }^{\mathrm{c}}$, Hiroshi Nakajima ${ }^{\mathrm{c}}$ \\ ${ }^{a}$ Himeji Initiative in Computational Medical and Health Technology, \\ Graduate School of Engineering, University of Hyogo, 2167 Shosha, Himeji, 671-2280, JAPAN \\ ${ }^{b}$ WPI Immunology Frontier Research Center, Osaka University, Suita, Osaka, 565-0871, JAPAN \\ 'Omron Corporation, 9-1, Kizugawadai, Kizugawa, Kyoto, JAPAN, 619-0283
}

\begin{abstract}
This paper proposes a heart rate monitoring system for detecting autonomic nervous system by the heart rate variability using an air pressure sensor to diagnose mental disease. Moreover, we propose a human behavior monitoring system for detecting the human trajectory in home by an infrared camera. In day and night times, the human behavior monitoring system detects the human movement in home. The heart rate monitoring system detects the heart rate in bed in night time. The air pressure sensor consists of a rubber tube, cushion cover and pressure sensor, and it detects the heart rate by setting it to bed. It unconstraintly detects the RR-intervals; thereby the autonomic nervous system can be assessed. The autonomic nervous system analysis can examine the mental disease. While, the human behavior monitoring system obtains distance distribution image by an infrared camera. It classifies adult, child and the other object from distance distribution obtained by the camera, and records their trajectories. This behavior, i.e., trajectory in home, strongly corresponds to cognitive disorders. Thus, the total system can detect mental disease and cognitive disorders by uncontacted sensors to human body.
\end{abstract}

Keywords: health care system, functional assessment of autonomic nervous system, human trajectory, Fuzzy Logic, heart rate variability, 3D infrared camera

\section{INTRODUCTION}

On March 11, 2011, the Tohoku area in Japan was hit by the biggest tsunami caused by a big earthquake. There were many fatalities, with survivors forced into temporary housing. Environmental and financial issues have continued to delay recovery. Survivors need a unique system to care and to prevent their mental disease. First, disaster survivors have a higher incidence of suicide, as shown by studies of the Hansin disaster in Japan, and need mental health care. Autonomic nervous system is the nervous system regulating an involuntary function such as breathing, sweat, the metabolism and the internal organs of the whole body. Autonomic nervous system is categorized as sympathetic system and parasympathetic system, and both nervous systems complementary work in human body. These nervous systems manage the human mind and body. Therefore, functional assessment of autonomic nervous system is useful to evaluate mental condition of a human. Heart rate variability (HRV)[1][2][3] is a recognized measure of autonomic control and is used as a clinical tool. Spectral analysis of HRV based on Fast Fourier Transform (FFT) shows that there are three major spectral regions. We are able to perform functional assessment of autonomic nervous system (ANS) for an index in these frequency components.

This paper first describes an autonomic nerve system assessment system to monitor mental health in disaster survivors using an air mat [4]-[8] incorporating sensors for heart rate. The used mat [9][10] has been used for functional assessment of the autonomic nervous system through heart rate variability. The mat consists of a sheet and a pressure sensor, and detects the biological information by setting under the bottom of a mattress. This sensor detects a pressure change by expanding and deflating of the tube in the mat. This mat is used on a bed or chair. We employ a detection method of heartbeat point using fuzzy membership functions [11]. This method improves the detection precision of the heartbeat by updating membership function with previous data. We evaluate the autonomic nervous system from a spectrum of the HRV. We evaluate it by comparing our result with truth result obtained by an electrocardiograph. In the result of our experiment on ten volunteers, we were able to perform functional assessment of autonomic nervous system with the HRV. This paper second describes a human trajectory detection system whose data contribute to diagnose

Independent Component Analyses, Compressive Sampling, Wavelets, Neural Net, Biosystems, and Nanoengineering X, edited by Harold Szu, Liyi Dai, Proc. of SPIE Vol. 8401, 840119 · @ 2012 SPIE · CCC code: 0277-786X/12/\$18 doi: $10.1117 / 12.918552$

Proc. of SPIE Vol. $8401840119-1$ 
mental diseases in survivors living in temporary housing. The used infrared three dimensional camera measures distance based on time of flight (TOF) principle. In it, the camera catches the shapes [12][13] in the housing, and directly delivers depth values for each pixel in real-time processing without the need of complex calculation algorithms and external reference points. This camera is also available in night. First, a human trajectory monitoring system classifies adult, child, and the other, and then detects the human behavior such trajectory of their movements. In it, we briefly describe the classification system using distance distribution image with fuzzy logic [14]. We can identify the trajectory of an adult, child or the others. Then, physicians can diagnose the mental disease by analyzing the results.

\section{ASSESSMENT SYSTEM OF AUTONOMIC NERVE SYSTEM}

\subsection{Method}

Our experimental system is shown in Fig. 1[9][10]. Our system is composed of a mat, an electrocardiograph a control device (ADInstruments Pty. Ltd., ML856) and a personal computer. The electrocardiograph is attached to the chest of the subject, and an mat is placed under the mattress. The mat consists of polypropylene sheet of $175 \mathrm{~mm} \times 780 \mathrm{~mm}$ and ultrasensitive pressure sensor (Fuji Ceramics Co., FKS-111) as shown in Figure 1(b). The sheet contains natural rubber tube of $5 \mathrm{~mm}$ (inside diameter) $\times 7 \mathrm{~mm}$ (outside diameter) $\times 3 \mathrm{~m}$ (length) and a cushion. The electrocardiograph needs for truth value measurement of heart rate.

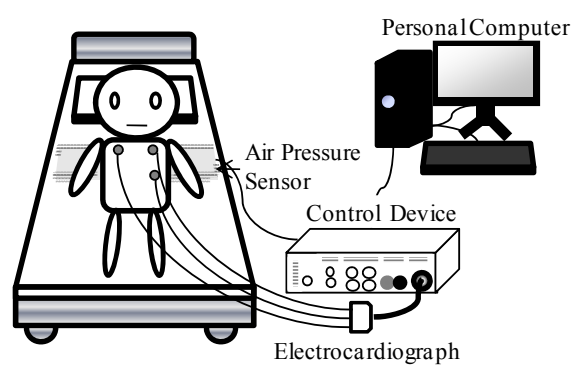

(a) Experiment setting in a bed or chair

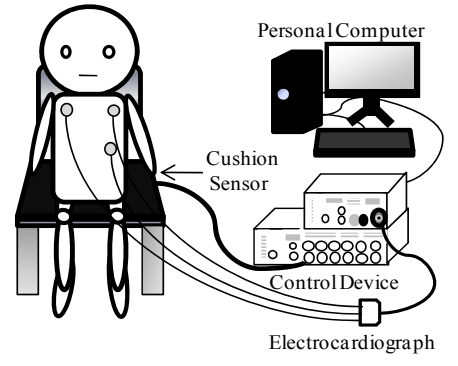

Figure 1. Experimental system.

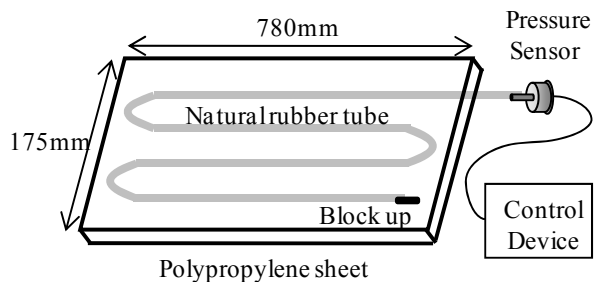

(b) Mat system

The ultrasensitive pressure sensor converts the air pressure change in the tube to an electronic signal, and outputs the signal based on $3 \mathrm{~V}$. This signal is quantized to 1024 levels (10bits) by a personal computer. The obtained data of the air pressure sensor and the electrocardiograph are provided to a personal computer. The sampling interval of the pressure sensor and the electrocardiograph are $2.5 \mathrm{msec}$.

HRV analysis is performed according to Ref. [1]. We use the method of short-term recordings of 2 to 5minutes. The spectrum of heart rate variability signal is calculated from tachogram of an interval of the heartbeat points. The interval of heartbeat points are defined as the RR intervals. The RR interval is an interval in time of $R$ wave which is the peak of the electrocardiograph. The RR interval tachogram is the RR intervals vs. the number of progressive beats, as shown in Figure 2. We employ FFT as the frequency analysis of the RR interval tachogram. In the frequency domain of the RR interval tachogram, three main spectral components of very low frequency (VLF), low frequency (LF), and high frequency (HF) are distinguished in a spectrum calculated from short-term recordings, as shown in Figure 3. The frequency range of VLF is less than or equal to $0.04 \mathrm{~Hz}$ and the frequency range of LF is 0.04 to $0.15 \mathrm{~Hz}$, and the frequency range of $\mathrm{HF}$ is 0.15 to $0.4 \mathrm{~Hz}$. In the control of the heartbeat change by the autonomic nervous system, the sympathetic system affects a change of low frequency, and the parasympathetic system affects a change of both frequency, but affects mainly a change of high frequency. Therefore frequency components of the factor of both the sympathetic system and parasympathetic are shown in LF, and frequency component of the factor of the parasympathetic is shown in HF. LF and HF are calculated in normalized units (n.u.) by Eqs. (1) and (2), respectively.

$L F($ n.u. $)=\frac{L F}{\text { TotalPower }-V L F} \times 100$ 
$H F($ n.u. $)=\frac{H F}{\text { TotalPower }-V L F} \times 100$

TotalPower in Eqs. (1) and (2) shows a frequency domain less than or equal to $0.4 \mathrm{~Hz}$. As mentioned above, LF is not only frequency component of the factor of the sympathetic system but also the frequency component of the factor of the parasympathetic system. Therefore, we regard LF/HF as an index of the sympathetic system, and we regard HF(n.u.) as an index of the parasympathetic system.

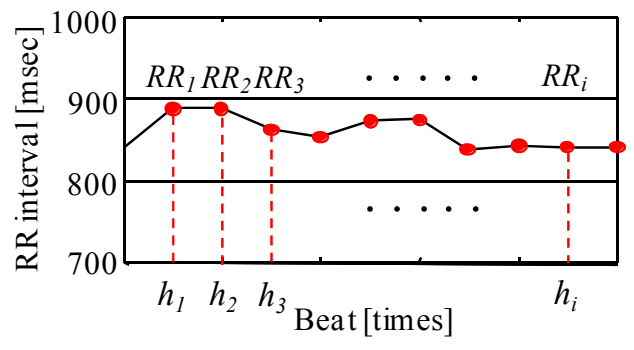

Figure 2 RR interval tachogram.

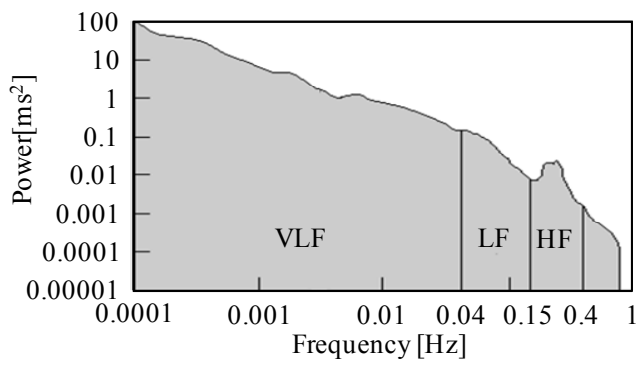

Figure 3 Example of spectrum density.

We detect the heart beat points which regarded as R-wave point below[9][10][14].

By using these properties, we propose a detection algorithm for biosignal points in the framework of fuzzy logic. From the above consideration, we can obtain the following knowledge.

Knowledge 1 : A large peak is the biosignal peak.

Knowledge 2 : The biosignal has no sudden change, it has a cycle.

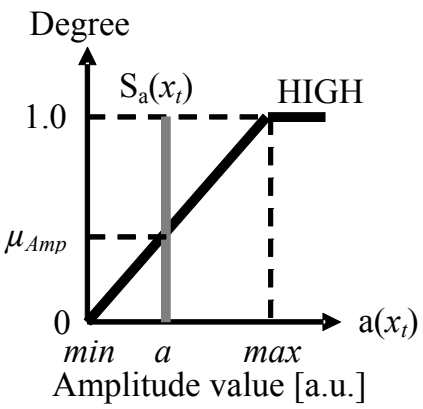

(a) Rule 1

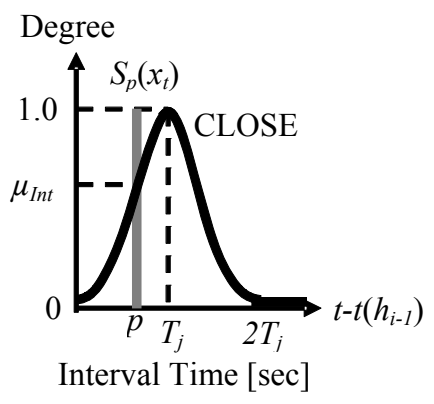

(b) Rule 2

Figure 4. Fuzzy membership functions for detecting heart signal. 
We explain a fuzzy detection algorithm on the basis of the above knowledge.

$<$ Fuzzy biosignal detection algorithm $>[14]$

For an input biosignal value $x_{t},(\mathrm{t}:$ time $)$, we denote the amplitude by $a\left(x_{t}\right)$. The notation $h_{i}(i=1,2, \ldots, \mathrm{n})$ denotes the biosignal peak point (target point).

Step 0: Set $i=1$, we determine the point of the first highest peak point as $h_{l}$.

Step 1: For $i \leftarrow i+1$, we define two fuzzy membership functions: one extracts the amplitude peak based on Knowledge 1 and the other extracts the period based on Knowledge 2. First, we derive the following fuzzy rules, where the notations $\mu_{A m p}\left(x_{t}\right)$ and $\mu_{I n t}\left(x_{t}\right)$ denotes the fuzzy degrees with respect to the amplitude and interval, respectively.

Rule 1 : IF $a\left(x_{t}\right)$ is HIGH, THEN the fuzzy degree of the bio-signal point, $\mu_{A m p}\left(x_{t}\right)$, is high.

Rule 2 : IF $t$ is CLOSE to the cycle $T_{j}$, THEN the fuzzy degree of bio-signal point, $\mu_{\text {Int }}\left(x_{t}\right)$, is high.

The fuzzy membership functions HIGH and CLOSE are defined in Figure 4. In Figure 4, the notation $T_{j}$ denotes a cycle of the biosignal. The determination method of $T_{j}$ is mentioned in next section. In Figure 4(a), the notations min and max are dynamic parameters which are calculated as the minimum and maximum values in the range between $\mathrm{t}\left(h_{i-1}\right)$ and $\mathrm{t}\left(h_{i-}\right.$ $\left.{ }_{1}\right)+2 \times T_{j}$ [sec], respectively. The notation $\mathrm{t}\left(h_{i}\right)$ denotes the time at $h_{i}$. In Figure 4(b), "CLOSE" membership function is calculated by Eq. (3) in the time range of $\mathrm{t}\left(h_{i-1}\right) \leq \mathrm{t} \leq \mathrm{t}\left(h_{i-1}\right)+2 \times T_{j}[\mathrm{sec}]$..

$\operatorname{CLOSE}\left(t-t\left(h_{i-1}\right)\right)=\exp \left(\frac{-\left(t-t\left(h_{i-1}\right)-T_{j}\right)^{2}}{2\left(T_{j} / 3\right)^{2}}\right)$

Fuzzy singleton functions are defined by Eqs. (4) and (5), respectively.

$s_{a}\left(x_{t}\right)= \begin{cases}1 & \text { if } a\left(x_{t}\right)=a \\ 0 & \text { otherwise }\end{cases}$

$s_{p}\left(x_{t}\right)=\left\{\begin{array}{cc}1 & \text { if } t-t\left(h_{i-1}\right)=p \\ 0 & \text { otherwise }\end{array}\right.$

We decide $h_{i}$ by using $h_{i-1}$ and $T_{i}$. The fuzzy degrees $\mu_{A m p}\left(x_{t}\right)$ and $\mu_{I n t}\left(\mathrm{x}_{t}\right)$ are calculated by Eqs. (6) and (7), respectively.

$$
\begin{aligned}
& \mu_{A m p}\left(x_{t}\right)=\min \left(H I G H, s_{a}\left(x_{t}\right)\right) \\
& \mu_{I n t}\left(x_{t}\right)=\min \left(C L O S E, s_{p}\left(x_{t}\right)\right)
\end{aligned}
$$

The total degree $\mu_{\text {Total }}\left(x_{t}\right)$ of the heartbeat point is calculated by the algebraic product by Eq. (8).

$\mu_{T}\left(x_{t}\right)=\mu_{\text {Amp }}\left(x_{t}\right) \times \mu_{\text {Int }}\left(x_{t}\right)$

We determine the bio-signal point $h_{i}$ as the point with the maximum $\mu_{T}\left(x_{t}\right)$.

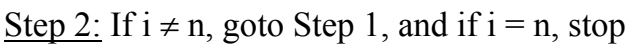

\subsection{Experimental Result}

In an experiment, we employed ten healthy male volunteers as shown in Table 1 . We measure the heart waves by electrocardiograph (ADInstruments Pty. Ltd., ML132) at the same time. We employ data of the electrocardiogram as the truth value. First, we show an experimental result in bed shown in Figure 1(a). We obtained data of five minutes for every subject. After we detect heart rate variability, we perform autonomic nervous system assessment by the HRV analysis. Table 2 shows the result of correlation coefficients of the HRV by our method. The average of correlation coefficients of HRV was 0.894 . Table 3 shows results of the autonomic nervous system evaluation. The average error of HF(n.u.) was $13.32 \%$, and the average error of LF/HF was $19.16 \%$. Second, we show an experimental result on a chair shown in Figure 1(a). The mat is placed on a chair and we evaluate accuracy of functional assessment of autonomic 
nervous system. We employed A-F volunteers in Table 1. We obtained data of five minutes for each. After we detect heart rate variability, we perform the HRV analysis. The average of correlation coefficients of HRV was 0.846 . Table 4 shows results of functional assessment of autonomic nervous system. The mean error of HF(n.u.) was $18.34 \%$, and the average error of LF/HF was $16.99 \%$. Consequently, they are enough to assess their autonomic nervous system.

Table 1 Subjects data.

\begin{tabular}{|c|c|c|c|}
\hline Subject & Age & Height $(\mathrm{cm})$ & Weight $(\mathrm{kg})$ \\
\hline \hline A & 23 & 180 & 95 \\
\hline B & 22 & 165 & 58 \\
\hline C & 22 & 175 & 61 \\
\hline D & 22 & 167 & 64 \\
\hline E & 21 & 179 & 67 \\
\hline F & 22 & 166 & 67 \\
\hline G & 23 & 172 & 57 \\
\hline H & 21 & 173 & 65 \\
\hline I & 22 & 160 & 40 \\
\hline J & 21 & 172 & 76 \\
\hline
\end{tabular}

Table 2 Result of correlation of HRV.

\begin{tabular}{|c|c|}
\hline Subject & Correlation Coefficient \\
\hline \hline A & 0.941 \\
\hline B & 0.916 \\
\hline C & 0.994 \\
\hline D & 0.890 \\
\hline E & 0.748 \\
\hline F & 0.833 \\
\hline G & 0.975 \\
\hline H & 0.990 \\
\hline I & 0.829 \\
\hline J & 0.828 \\
\hline
\end{tabular}


Table 3 Result of ANS assessment in bed.

\begin{tabular}{|c|c|c|c|c|}
\hline \multicolumn{2}{|c|}{ Subject } & Our method & Truth Value & Error (\%) \\
\hline \hline \multirow{2}{*}{$A$} & HF(n.u.) & 48.29 & 43.51 & 10.99 \\
\cline { 2 - 5 } & LF/HF & 1.07 & 1.29 & 17.05 \\
\hline \multirow{2}{*}{$B$} & HF(n.u.) & 45.88 & 42.07 & 9.06 \\
\cline { 2 - 5 } & LF/HF & 1.18 & 1.37 & 13.87 \\
\hline \multirow{2}{*}{ C } & HF(n.u.) & 80.71 & 81.70 & 1.21 \\
\cline { 2 - 5 } & LF/HF & 0.24 & 0.22 & 6.70 \\
\hline \multirow{2}{*}{ D } & HF(n.u.) & 32.48 & 21.96 & 47.91 \\
\cline { 2 - 5 } & LF/HF & 2.08 & 3.54 & 41.24 \\
\hline \multirow{3}{*}{$E$} & HF(n.u.) & 56.57 & 50.87 & 11.21 \\
\cline { 2 - 5 } & LF/HF & 0.77 & 0.97 & 20.41 \\
\hline \multirow{2}{*}{$F$} & HF(n.u.) & 69.72 & 69.87 & 0.21 \\
\cline { 2 - 5 } & LF/HF & 0.43 & 0.44 & 1.63 \\
\hline \multirow{2}{*}{ G } & HF(n.u.) & 45.87 & 51.01 & 10.08 \\
\cline { 2 - 5 } & LF/HF & 1.18 & 0.97 & 21.65 \\
\hline \multirow{2}{*}{$H$} & HF(n.u.) & 47.07 & 46.21 & 1.86 \\
\cline { 2 - 5 } & LF/HF & 1.12 & 1.16 & 3.45 \\
\hline \multirow{2}{*}{ I } & HF(n.u.) & 65.90 & 49.68 & 32.65 \\
\cline { 2 - 5 } & LF/HF & 0.52 & 1.01 & 48.81 \\
\hline \multirow{2}{*}{$J$} & HF(n.u.) & 60.44 & 55.97 & 7.99 \\
\cline { 2 - 5 } & LF/HF & 0.65 & 0.79 & 16.79 \\
\hline \multirow{2}{*}{} & & & \\
\hline
\end{tabular}

Table 4 Results of ANS assessment on chair

\begin{tabular}{|c|c|r|r|r|}
\hline \multicolumn{2}{|c|}{ Subject } & Our method & Truth Value & Error(\%) \\
\hline \hline \multirow{2}{*}{ A } & HF(n.u.) & 30.97 & 30.76 & 0.68 \\
\cline { 2 - 5 } & LF/HF & 2.23 & 2.25 & 0.89 \\
\hline \multirow{2}{*}{ B } & HF(n.u.) & 69.61 & 67.88 & 2.55 \\
\cline { 2 - 5 } & LF/HF & 0.44 & 0.47 & 6.38 \\
\hline \multirow{2}{*}{ C } & HF(n.u.) & 86.16 & 87.16 & 1.15 \\
\cline { 2 - 5 } & LF/HF & 0.16 & 0.15 & 6.67 \\
\hline \multirow{2}{*}{ D } & HF(n.u.) & 45.90 & 24.10 & 90.46 \\
\cline { 2 - 5 } & LF/HF & 1.18 & 3.14 & 62.42 \\
\hline \multirow{2}{*}{ E } & HF(n.u.) & 60.37 & 59.92 & 0.75 \\
\cline { 2 - 5 } & LF/HF & 0.66 & 0.67 & 1.49 \\
\hline \multirow{2}{*}{ F } & HF(n.u.) & 55.02 & 48.08 & 14.43 \\
\cline { 2 - 5 } & LF/HF & 0.82 & 1.08 & 24.07 \\
\hline
\end{tabular}




\section{HUMAN TRAJECTORY MONITORING IN HOUSE}

Human trajectory provides information with respect to mental disease. For example, if a subject is in a room whole day, we must suspect some mental disease. If a subject frequently goes to bath room in night, we must suspect dementia. In our study, we use the 3D camera SwissRanger SR-4000, MESA imaging. This camera measures the distance based on phase-measuring TOF principle and acquires the distance distribution as depth map image. Figure 5 shows a gray level image and the three dimensional image. This camera measures the distance for the range from $0.0 \mathrm{~m}$ to $5.0 \mathrm{~m}$, which is quantized to 65536 levels (16-bits). The image resolution is $176 \times 144$ pixels. The proposed method identifies the objects by preprocessing and clustering process from distance distribution image, and we make fuzzy if-then rules from knowledge of adult, child and the other object so as to classify them[12][13]. Here, we make the fuzzy membership functions with respect to the features based on the human characteristics like height, thickness, and so on. We calculate the fuzzy degree of adult, child and object from these features and thereby we decide it to one of adult, child and the other object.
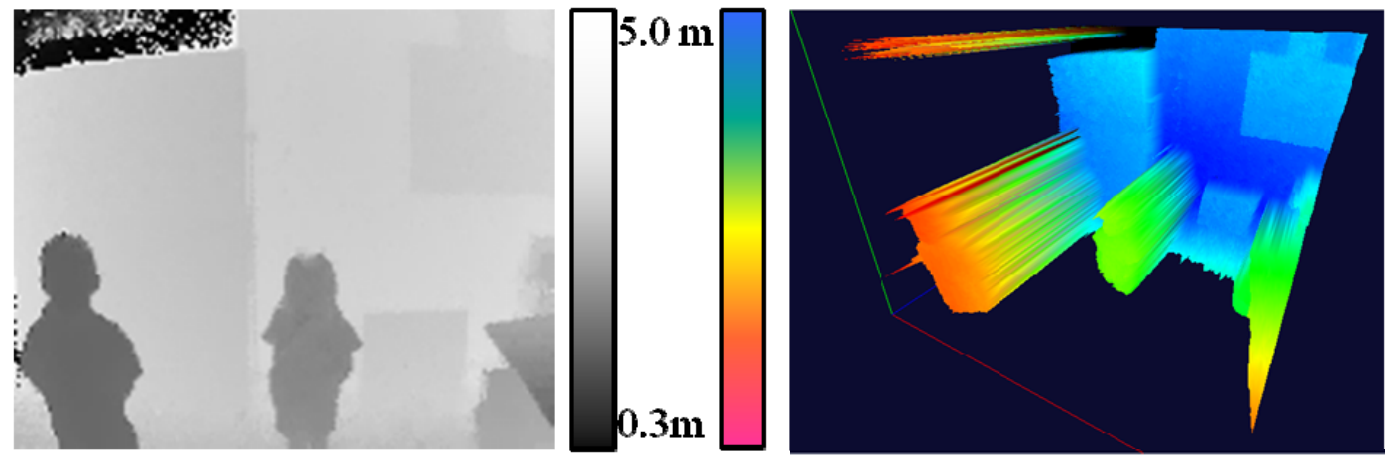

Figue 5 Gray level image and 3 dimentional imag.

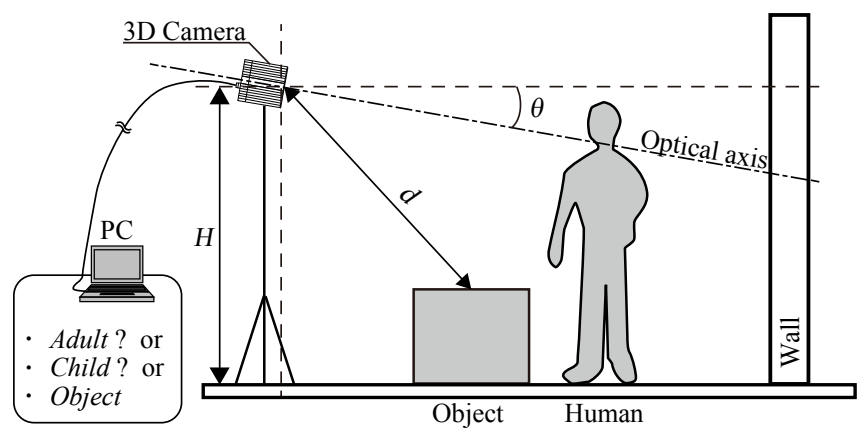

Figure 6. Experimenal system.

Figure 6 shows our experimental system. The camera measures distance distribution. We set the 3D camera which installation parameters were $\mathrm{H}=1.6 \mathrm{~m}$ and $\theta=10^{\circ}$. Our method consists of four steps [13]. The summary of this method is as follows.

Step 1: We remove the background except for human, object and animal to extract the moving objects from distance distribution image.(see Figures 7 and 8)

Step 2: We remove noise and do clustering of the image, and we get a binary image. (see Figures 9 and 10) 
Step 3: We extract features such as the height, thickness, aspect ratio and area ratio. Then, we make fuzzy if-then rules from knowledge of adult, child and the other object. Here, we make the fuzzy membership functions with respect to each feature.

Step 4: We classify the clusters to adult, child and the other object by using these fuzzy degrees and record their trajectories

Please refer the detailed method in Ref.[13]. The experiment was done for 10 seconds, i.e, 300 flames. The method classified human with the accuracy in Table 5. Table 6 tabulated the accuracy of tall measurement.

Table 5 Experimental results in human classification.

\begin{tabular}{|c|c|c|c|}
\hline ID \# & age & tall(m) & Accuracy (\%) \\
\hline \#A & 4 & 0.98 & $97.2 \%$ \\
\hline \#B & 5 & 1.11 & $97.1 \%$ \\
\hline \#C & 7 & 1.33 & $98.8 \%$ \\
\hline \#D & 9 & 1.31 & $99.6 \%$ \\
\hline \#E & 21 & 1.55 & $100.0 \%$ \\
\hline \#F & 23 & 1.60 & $100.0 \%$ \\
\hline \#G & 22 & 1.72 & $100.0 \%$ \\
\hline
\end{tabular}

Table 6 Tall measurement results.

\begin{tabular}{|c|c|c|c|}
\hline ID \# & True value $(\mathrm{m})$ & Our method $(\mathrm{m})$ & error \\
\hline \#A & 0.980 & 0.932 & -0.048 \\
\hline \#B & 1.110 & 1.068 & -0.042 \\
\hline \#C & 1.330 & 1.247 & -0.083 \\
\hline \#D & 1.310 & 1.246 & -0.064 \\
\hline \#E & 1.540 & 1.543 & 0.003 \\
\hline \#F & 1.600 & 1.650 & 0.050 \\
\hline \#G & 1.720 & 1.786 & 0.066 \\
\hline \multicolumn{4}{|c|}{ Mean absolute error } \\
\hline
\end{tabular}




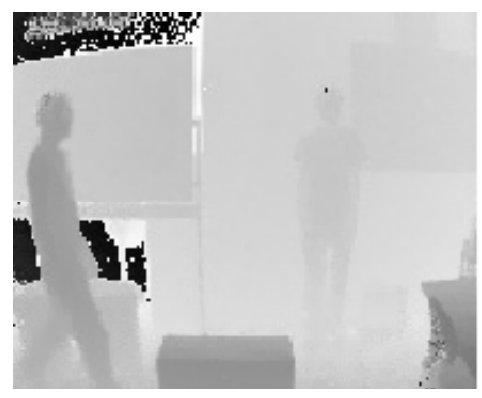

Figure 7 A raw image.

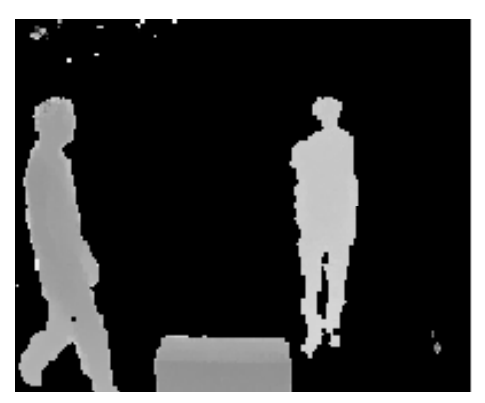

Figure 8 Removing background.

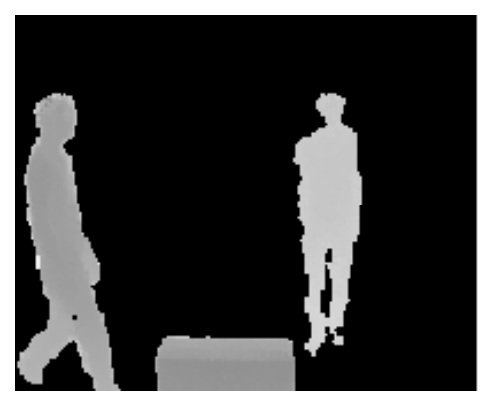

Figure 9 Noise reduction.

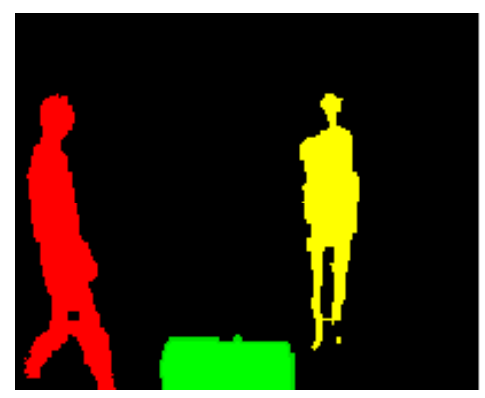

Figure 10 Clustering. 


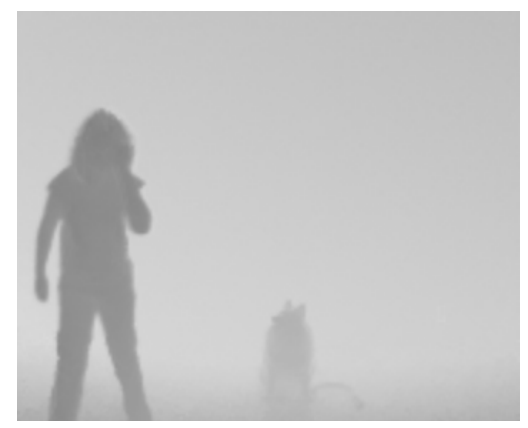

Figure 11 Adult woman and dog.

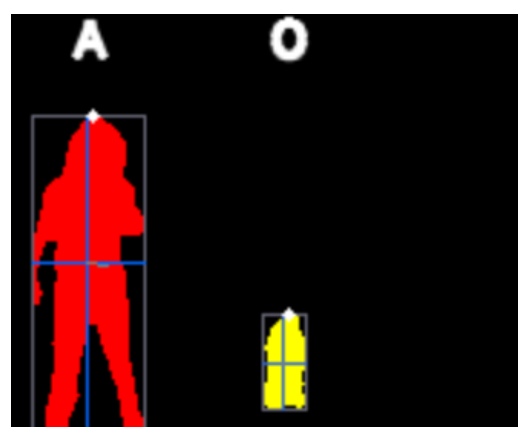

Figure 12 classification of Figure 11.

In our experiment, the proposed method classified human and a dog. Figure 11 shows a resultant example frame of classification. Figure 12 shows the result of Figure 11. The mean accuracy rate between a human and a dog was $100 \%$. After we classify them, we can easily analyze each trajectory of movement by recording each center of gravity of the classified objects for every frame. Figure 13 shows an example image of trajectory. Blue, red and green lines shows trajectory. In them we can identify one of the adult, child or dog. Thus, we can see the human movement, and then medical doctors can analyze the results.

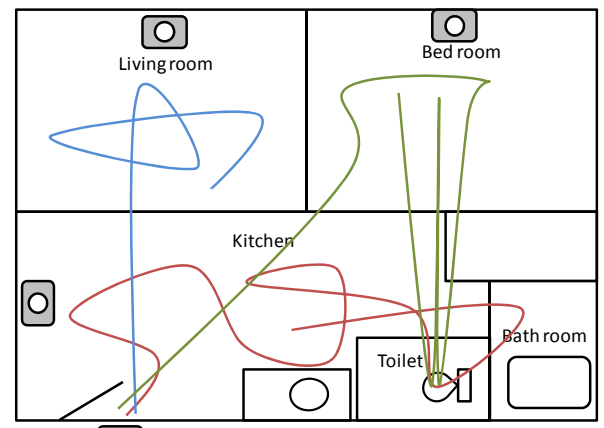

O) camera

Figure 13 Images of trajectory of temporary houses.

\section{CONCLUSIONS}

This paper has described an autonomic nerve system assessment system to monitor mental health using an air mat incorporating sensors for heart rate. The used mat has been used for functional assessment of the autonomic nervous 
system through heart rate variability. The mat system achieved high precision and low cost measurement. This mat is useful to the monitoring on a bed or chair without no direct body contact. Non contacted sensing system is a promising direction to 24 hour sensing system. Its software employed fuzzy membership functions with dynamic parameters; thereby it achieved high accuracy with respect to functional assessment of autonomic nervous system with the HRV. This study would be one of low cost and high accuracy sensing systems [15][16] of HRV analysis. Secondly, this paper has described a human trajectory detection system whose data contribute to diagnose mental diseases in house. In it, a three-dimensional (3D) distance measuring camera was used. Although this camera is high cost, it identifies an adult, child, animal and the others by catching the object shapes in the house, and thereby we can identify the trajectory of every subject. Then, physicians can diagnose the mental disease by analyzing the results. If we can solve the privacy problem with respect to camera usage, it would be the best way to monitor them. We must develop a method with low cost and lower resolution enough to keep the privacy.

\section{REFERENCES}

[1] Malik, M., "Heart Rate Variability - Standards of Measurement, Physiological Interpretation, and Clinical Use," Annals of Noninvasive Electrocardiology, 1( 2), 151-181 (1996)

[2] Berger, R. D., Akselrod, S., Gordon, D. and Cohen, R. J., "An efficient algorithm for spectral analysis of heart rate variability,” IEEE. Transactions on Biomedical Engineering, BME-33(9), 900-904 (1986).

[3] Arts, C. V. R., Kollee, L. and Hopman, J. "Heart rate variability,” Ann Intern Med. 118, 436-447 (1993).

[4] Kamozaki, Y., Sawayama, T., Taniguchi, K., Kobashi, S., Kondo, K. and Hata, Y., "Fuzzy Extraction System for Heart Pulse by Air Pressure Sensor," Proc. International Symposium on Intelligent Signal Processing and Communication Systems, 919-922 (2006).

[5] Hata, Y., Kamozaki, Y., Sawayama, T., Taniguchi, K., and Nakajima, H., "A Heart Pulse Monitoring System by Air Pressure and Ultrasonic Sensor Systems," Proc. IEEE System of Systems Engineering, 1-5, 2007.

[6] Hata, Y., Yamaguchi, H., Kobashi, S., Taniguchi, K., and Nakajima, H., "A Human Health Monitoring System of Systems in Bed," Proc. IEEE Int. Conf. on System of Systems Engineering, 1-5 (2008).

[7] Hata Y., Kobashi S., Taniguchi K., and Nakajima H., "Human health monitoring system of systems with fuzzy logic by sensor network," Proc. IEEE Workshop on Robotic Intelligence in Informationally Structured Space, 16 (2009).

[8] Hata, Y., Kobashi, S., Yamaguchi, H., Ishikawa, O., Tsuchiya, N., and Nakajima, H., "Human health monitoring system of systems by non-contacted sensors," Proc. IEEE Int. Conf. on System of Systems Engineering, 1-5 (2009).

[9] Yamamoto, K., Kobashi, S., Hata, Y., Tsuchiya, N. and Nakajima, H., "Fuzzy Heart Rate Variability Detection by Air Pressure Sensor for Evaluating Autonomic Nervous System," Proc. IEEE International Conference on Systems, Man, and Cybernetics, 3067-3071 (2008).

[10] Yamamoto, K., Kobashi, S., Hata, Y., Tsuchiya, N., and Nakajima, H., "Real time autonomic nervous system display with air cushion sensor while seated," Proc. IEEE Int. Conf. on Systems, Man and Cybernetics, 11161121 (2009).

[11]Zadeh, L.A., [Fuzzy Sets and Applications], New York: John Wiley and Sons, (1987).

[12] Ho, K., Taniguchi, K., Asari, K., Kuramoto, K., Kobashi, S., and Hata, Y., "Automated Detection of People Distribution by a 3D Camera," Proc. World Automation Congress, 1-6, (2010).

[13] Kanazawa, S., Taniguchi, K., Kazunari, A., Kuramoto, K., Kobashi, S. and Hata, Y., "A fuzzy automated object classification by infrared laser camera," Proc. SPIE Defense, Security and Sensing, 805815, 1-9 (2011).

[14]Hata, Y., Kobashi, S., Kuramoto, K., and Nakajima, H., "Fuzzy Biosignal Detection Algorithm and Its Application to Health Monitoring," International Journal of Applied and Computational Mathematics, 10(1), 133-145 (2011).

[15] Ishijima, M., "Monitoring of Electrocardiograms in Bed without Utilizing Body Surface Electrodes," IEEE Transactions on Biomedical Engineering, 40(6), 593-594, (1993).

[16] Watanabe, K., Watanabe, T., Ando, H., Ishikawa, T., and Kobayashi, K., "Noninvasive Measurement of Heartbeat, Respiration, Snoring and Body Movement of a Subject in Bed via a Pneumatic Method", IEEE Trans. on Biomedical Eng. 52(12), 100-2107 (2005). 